\title{
The Business Case: Collaborating to Help Employees Maintain Their Mental Well-Being $\odot$
}

INVITED ESSAY

Sari Sairanen

National Health and Safety Director

CAW Canada

Deanna Matzanke

Director, Global Employment Strategies

Global Human Resources and Communications, Scotiabank

Doug Smeall

Assistant Vice-President, Health Management Services

Sun Life Financial

$\propto$

ABSTRACT

There has been a change in the mindset of businesses in recent years. Companies are starting to realize that proactively helping their employees to maintain mental health is beneficial, both for their workers and their business. In this article, we present three different but complementary views - those of an advocate, an employer and a provider - on helping employees maintain mental, and physical, health. In the first section, Sari Sairanen outlines programs and services to manage stress and maintain mental health that have been developed by the Canadian Auto Workers' union and implemented in partnership with employers, wellness providers, service agencies and other community partners. The union focuses on raising awareness and providing education, as well as removing the stigma associated with mental illness. Deanna Matzanke, in her section, discusses the commitment of a company, Scotiabank, to create and maintain an inclusive and accessible workplace for all its employees. It has recently worked with providers to develop and implement integrated services dealing 
The Business Case: Collaborating to Help Employees Maintain Their Mental Well-Being

specifically with mental health illness and addiction, which aid not only its current employees but also possible future employees. Finally, Doug Smeall shares his observations as an insurer at Sun Life Financial, who has seen the rates of both shortterm and long-term disabilities increase. He elaborates on the collaborative work between insurers and employers to help employees maintain their mental health, and to return to work sooner when issues do occur. Ultimately, this article argues that unions, employers and insurers can work together with partners and employees to promote and maintain employee health because, as Sairanen asserts, "preventing a problem in the first place is the best strategy."

\section{We Can Do It: Evidence and Interventions for Transforming Mental Health in the Workplace}

The Canadian Auto Workers' union (CAW) is the largest private sector union in Canada, with over 225,000 members from coast to coast. Our members work in aerospace, mining, fishing, auto and specialty vehicle assembly, auto parts, hotels, airlines, rail, education, hospitality, retail, road transportation, healthcare, manufacturing, shipbuilding and other sectors of the economy. CAW is not only dedicated to fighting for workers' rights at the bargaining table, it is equally committed to taking on economic, political and social issues that affect its members and their families in the broader community.

As a result, we have and continue to focus on developing new and innovative strategies for creating a healthy supportive workplace culture. One of the ongoing challenges in this regard is the amount of stress that members experience. In the present state of the economy, more than ever, tremendous pressure is being put on workers, retirees and families concerning job security, productivity and pensions. This stress is on top of that normally associated with day-to-day living.

While those with good coping skills can manage some of this anxiety, for many the additional worry is too much. Pressure and mental strain mount over time, often resulting in burnout or illness. Even those who can normally deal with increased amounts and sources of stress can find themselves experiencing some of the social and health problems associated with unmanaged anxiety.

CAW has always believed that preventing a problem in the first place is the best strategy. We also feel that it is important to provide our members and their families with the tools and personal resources in their workplace and communities to assist them in dealing with challenges. In our initiatives, we promote both physical and mental health. We focus on increasing our members' understanding of mental health as a risk factor for chronic disease. Health is more than just the absence of disease.

When it comes to stress and overall mental health, CAW has been focused on activities such as raising awareness, education, skill building, early detection and illness management and integration strategies. Programs and services regarding stress and overall mental health have been developed and implemented in partnership with employers, wellness providers, service agencies and other community partners.

Despite mental health claims being the fastest-growing category of disability costs in Canada, it has been a challenge to convince our employer groups to embrace wellness programs. However, perseverance and the alignment of physical and mental health for better health outcomes and cost-savings for companies have 
helped to start conversations. In addition, CAW has used an approach of mental health promotion, focusing on the strengths, assets and capacities of employees rather than having a problem- or deficit-based orientation.

A clear message was given to our employer groups: CAW takes mental health in the workplace seriously. In the mid-1990s, we successfully bargained wellness programs within the auto industry; Motoring to Wellness at General Motors (GM), Working toward Wellness at Chrysler and Drive to Wellness at Ford. The common goals were to improve the health and well-being of employees, retirees and their families, to have working wellness committees and to implement comprehensive wellness programming via a variety of modalities.

\section{A clear message was given to our employer groups: CAW takes mental health in the workplace seriously.}

The joint wellness committees with the expert help of the Windsor-Essex County and Durham Region Health Units establish annual wellness goals for their respective workplaces. Each theme-based program consists of 12 core components such as health issues magazines, personal health guides, e-bulletins, contests, e-presentations, kick-off wellness events, posters and environmental and policy supports to provide multiple learning options.

Specifically, to support the mental health initiatives, the joint wellness committees developed programs such as Making Healthy Choices, Stress Management, Work-Life Balance and Sleep On It that were delivered using the core components in the workplaces. In addition, videos on aspects of mental health were produced and implemented into a regular health and safety routine. These videos addressed issues regarding workplace and home life and tips to reduce stress, such as positive self-talk, exercise and healthy eating, practising gratitude, getting adequate sleep, monitoring caffeine intake and "getting over stuff."

At GM, two- to five-minute videos depicting stress and depression vignettes were produced and piloted at one of the work locations to raise awareness and demystify mental illness. The initiative was so well received that the videos are now shown at all the GM work locations.

Unfortunately, the stigma of mental illness is a real obstacle in our workplaces. During a recent leadership meeting, a resolution regarding mental health was presented and debated by the more than 800 delegates from across Canada. Numerous delegates lined up at the microphones and passionately shared personal stories and encouraged the adoption of the resolution, which was unanimously passed:

Therefore be it resolved that the National CAW develops a strategy to understand the scope and effects of mental illness on our membership ...

And be it further resolved that the CAW National Health and Safety Department develop and implement a national awareness campaign to educate all its members on mental illness, thereby remove the stereotype and stigma associated with this illness.

Sigmund Freud once said, "Love and work are the cornerstones of our humanness." Freud was perhaps one of the first to recognize the connection between work and mental health. Since his time (1856-1939), a lot of research has shown that work is important, if not essential, to a person's mental well-being. Mental disabilities are often challenging to identify and respond to appropriately in the workplace. 
What research and experience have shown is that we can make a difference in our workplaces through effective leadership, education and training, communication to reduce the stigma, early intervention and, most of all, collective efforts and shared learning.

- Sari Sairanen

\section{Mental Health Wellness in the Workplace: Partnering for the 21st Century}

During its 178-year history, Scotiabank has made many strides in the area of inclusion of persons with disabilities in the workplace. Those strides have, in large part, been the result of a sense of commitment by managers and leaders across the bank to reflect the communities in which they serve and to do the right thing in welcoming historically disadvantaged groups into the workplace. In the vast majority of individual cases, the bank has been successful. However, these efforts have typically happened spontaneously or reactively in the spirit of addressing an immediate challenge or issue and have not generally been driven by a clear, proactive strategy to identify, address and eliminate barriers to or in the workplace for persons with disabilities and, in particular, those with a mental health illness or addiction.

Recognizing that the health and wellbeing or our employees is of paramount importance, and understanding that a coordinated or integrated effort was needed to effectively address some of the rising costs of mental health illness in the workplace, Scotiabank introduced a Wellness Strategy in 2004. Under this strategy, the bank focused on four key directions:

1. To incorporate wellness into policy, making it part of the fabric of who we are and what we do
2. To engage and challenge providers to integrate their services and offerings - creating an interconnectivity of physical and mental health and therefore wellness

3. To continue to achieve excellence in diversity, inclusivity and work-life balance

4. To offer integrated services and supports

Recently, the bank introduced a Global Diversity and Inclusion Strategy, which makes the commitment to create and maintain an inclusive and accessible workplace for all its employees and recognizes diversity and inclusion as important keys to business sustainability. Supporting this has been a long-standing and comprehensive flexible work options policy, as well as an accommodation policy with a centralized budget to support work adjustments and to purchase equipment.

\section{A coordinated or integrated effort was needed to effectively address some of the rising costs of mental bealth illness in the workplace.}

The key connecting thread between these two strategies is a focus on developing and maintaining key partnerships with our service providers, our employees, community organizations and subject matter experts and to actively encourage the integration of services and offerings to and for our employees - in short, partnering for the 21st century for the benefit of our employees and for Scotiabank. The following is a brief description of some of the key partnerships and integrated services and supports that have clearly filled a gap for many employees over the past few years.

Two important service providers for Scotiabank are our Employee Assistance Program (EAP) provider, Shepell-fgi, and our insurance provider, Great West Life. These 
companies have assisted us in developing and implementing new and integrated services dealing specifically with mental health illness and addiction.

Mental Health First Aid for Managers is a course offered through our EAP program that is available for any manager. This course addresses the signs of mental health issues and equips people leaders with the tools to address these problems as and when they arise in the course of business.

\section{The majority of closed cases reported a 50-74\% positive change in functionality.}

Depression Care, also provided through EAP, is an enhanced service that builds on basic confidential counselling services. It includes an extensive clinical assessment to determine the level of depression and the requirement for additional care.

EAP also offers other supportive offerings and services that include mind-body wellness, anger management, health coaching and a Health Assistance Program that includes Best Doctors - access to world-renowned doctors to ensure the accuracy of diagnosis and treatment.

Work Assist is an award-winning partnership between Scotiabank, Great West Life and Shepell-fgi. It is a voluntary counselling support service offered through the EAP that primarily focuses on emotional and mental health and is designed to help employees on short- or long-term disability benefits return to healthy and productive living. Most notably, the program is particularly helpful for those employees who are at work but who may be struggling with stress, anxiety or depression. Results from the program show that, in both 2008 and 2009, the majority of the closed cases were related to mental health issues. The best indicator that the program has been successful is the percentage of functional change in these cases - an increase in the persons' ability to function in daily living activities, including work. For both 2008 and 2009 , the majority of closed cases reported a $50-74 \%$ positive change in functionality. This is quite significant and has clear implications in terms of the effects on work productivity, both in quantity and quality.

Aside from key partnerships with our service providers, Scotiabank cultivates an ongoing relationship with its employee resource group, called Scotiabankers for Universal Access. The group's mandate is to provide a voice for employees with respect to systemic barriers in the workplace. Most recently, at the request of the Human Resources Department, the group facilitated efforts to provide a peer supporter for an employee who was struggling to deal with his work and a mental health illness.

Scotiabank is also fortunate to be closely involved with the work of several community organizations, including the Job Opportunity Information Network (JOIN), which focuses on the integration of persons with disabilities into the workforce; the Episodic Disability Initiative, through the Canadian Working Group on HIV and Rehabilitation (CWGHR); and the Toronto District School Board (TDSB) Employment Accessibility Exchange, which offers a financial corporate practice firm where candidates with disabilities can practise key work behaviours before entering the workforce.

Another key community partner is the Centre for Addiction and Mental Health (CAMH) Employment Support and Development program, which assists people who have mental health and addiction histories to secure, retain and advance 
in employment opportunities. The program offers counselling, skills training and ongoing professional support. In this case, Scotiabank has focused on helping CAMH identify candidates who can be referred to the TDSB practice firm. Once a candidate has reached a comfortable working level, he or she is referred for a position at Scotiabank. If hired, the new employee's manager undergoes our Mental Health First Aid for Managers course, and the new employee is connected with Scotiabankers for Universal Access. We also have plans to develop job aids for our managers on managing episodic disabilities, with the information and input from CAMH, JOIN and CWGHR.

Although these programs and partnerships are very new, all of those involved employees, managers, service providers and community partners - understand that what will make a difference to the inclusion of persons with mental health illness or addiction in the workplace is this kind of integration and partnering that provides a "warm hand-off" of talented candidates to other services that work in concert. The impact of each service or effort, although valuable on its own, is trebled in many cases, and together they create a consistent, linked and durable support line for a prospective employee, while managing an unpredictable illness. Internally, Work Assist, EAP and an employee resource group provide us with that same support line for existing employees. The key is partnering - partnering from a 21 st-century view of the globalized economy and social network in which we live and do business today.

- Deanna Matzanke

\section{An Insurer's Perspective}

Over the past several years, insurance companies have noticed a fairly steady increase in the percentage of short-term disability (STD) and long-term disability (LTD) claims that have a mental health condition listed as the primary or secondary diagnosis. It is not uncommon for the incidence of mental health issues for STD claims to be in the 30-40\% range for some clients (companies). For most clients, the prevalence of mental health conditions is approaching $30 \%$ of all LTD cases and has been increasing at a rate of approximately $0.5-1 \%$ per year over the past several years.

We are also observing high rates of co-morbidity of mental health conditions associated with such physical conditions as diabetes, cancers and cardiovascular, musculoskeletal and gastrointestinal disorders. The presence of mental health conditions as either a primary or secondary diagnosis is having an impact upon both the incidence and duration of STD and LTD cases.

Whereas a number of years ago, mental health conditions were more prevalent in traditionally white-collar industries, we are now witnessing greater reporting of mental health issues in blue- and grey-collar industries as well. It would appear that the stress on increasing productivity in all sectors is manifesting itself in a greater reporting of mental health events.

We are also witnessing the impact of the "treadmill" effect on rising rates of presenteeism, absenteeism and STD and LTD claims. Employees who feel under constant demands to produce and who are challenged to balance work and home pressures are filing STD and LTD claims in increasing numbers. This effect is compounded if the employees feel they lack the ability to control their workload or have limited authority to make decisions.

The average STD claim lasts between six and eight weeks, and the average LTD claim payment is in excess of $\$ 80,000$. The good news, however, is that we are finding that organizations are taking more proactive steps to prevent both physical and mental healthrelated disabilities from occurring in the 
workplace. More and more organizations are raising awareness of mental health issues at work, and they are making significant strides in reducing the associated stigma. A number of organizations have also moved beyond just raising awareness to implementing programs that engage employees and help to promote better physical and mental health. With the right amount of communication and by providing various incentives, they have been able to achieve significant rates of employee participation in these programs. They are recognizing that addressing organizational culture issues and providing healthy lifestylepromotion programs are starting to have an impact on the health and well-being of their people. Companies are seeing a reduction in the prevalence of health risk factors in their populations and are confident they will soon be able to measure reductions in the incidence and duration of absences from work.

\section{Organizations are raising awareness of mental health issues at work and are making significant strides in reducing the associated stigma.}

At Sun Life, we have witnessed an increased uptake of both our health assessment tools and our health promotion programs, which target both mental and physical health. Employers are leveraging online assessment and health promotion programs to assist their people in evaluating their overall state of health and adopting healthier lifestyle behaviours. We are also finding that organizations are working more closely with insurers to help their employees navigate the healthcare system and engage their healthcare teams and
EAP providers in proactively managing their health and increasing their levels of resiliency.

Health promotion programs (both online and in person) are also proving effective in helping employees recover from mental and physical health issues and return to work. At the same time, insurers have evolved their processes in recognition of the fact that a return to work after a mental health issue requires a different approach than one involving a solely physical cause.

Insurers have also developed training programs that are available to managers to help them to identify employees possibly struggling with a mental health issue. These programs additionally assist employees to receive help through their EAP provider or physician.

Organizations are finding that if they address organizational culture issues that are impacting on their employees' mental health and if they actively promote health assessment and promotion programs, they positively impact employee morale, productivity and engagement. They also impact rates of absenteeism and shorten the duration of both STD and LTD claims. Ultimately, if organizations sustain their levels of support for health assessment and health promotion programs, we expect that they will start to see reductions in the incidence of employee absence from work.

- Doug Smeall 